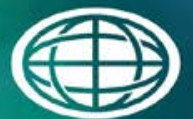

Savannah River

National Laboratory ${ }^{m}$

OPERATED BY SAVANNAH RIVER NUCLEAR SOLUTIONS

\title{
488-4D Ash Landfill Closure Cap HELP Modeling
}

Mark A. Phifer

November 2014

SRNL-STI-2014-00392, Revision 1 


\section{DISCLAIMER}

This work was prepared under an agreement with and funded by the U.S. Government. Neither the U.S. Government or its employees, nor any of its contractors, subcontractors or their employees, makes any express or implied:

1. warranty or assumes any legal liability for the accuracy, completeness, or for the use or results of such use of any information, product, or process disclosed; or

2. representation that such use or results of such use would not infringe privately owned rights; or

3. endorsement or recommendation of any specifically identified commercial product, process, or service.

Any views and opinions of authors expressed in this work do not necessarily state or reflect those of the United States Government, or its contractors, or subcontractors.

\section{Printed in the United States of America \\ Prepared for U.S. Department of Energy}


Keywords: Ash Landfill

Modeling

Retention: Permanent

\section{8-4D Ash Landfill Closure Cap HELP Modeling}

Mark A. Phifer

November 2014

Prepared for the U.S. Department of Energy under contract number DE-AC09-08SR22470. 


\section{REVIEWS AND APPROVALS}

AUTHOR:

Mark A. Phifer, Radiological Performance Assessment, SRNL

Date

TECHNICAL REVIEWER (Reviewed per E7 2.60):

Frank G. Smith, Radiological Performance Assessment, SRNL

Date

APPROVALS:

David A. Crowley, Manager

Date

Radiological Performance Assessment, SRNL

Robert S. Aylward, Manager

Date

Environmental Restoration Technology, SRNL

Tom F. Gaughan, Manager

Date

Area Completion Engineering, SRNS 


\section{ACKNOWLEDGEMENTS}

Tad Whiteside provided invaluable help by producing a virtual XP machine, which allowed the Hydrologic Evaluation of Landfill Performance (HELP) computer code to be run on a Windows 7 machine. 


\section{EXECUTIVE SUMMARY}

At the request of Area Completion Projects (ACP) in support of the 488-4D Landfill closure, the Savannah River National Laboratory (SRNL) has performed Hydrologic Evaluation of Landfill Performance (HELP) modeling of the planned 488-4D Ash Landfill closure cap to ensure that the South Carolina Department of Health and Environmental Control (SCDHEC) limit of no more than 12 inches of head on top of the barrier layer (saturated hydraulic conductivity of no more than $1.0 \mathrm{E}-05 \mathrm{~cm} / \mathrm{s}$ ) in association with a 25-year, 24-hour storm event is not projected to be exceeded. Based upon Weber 1998 a 25-year, 24-hour storm event at the Savannah River Site (SRS) is 6.1 inches. The results of the HELP modeling indicate that the greatest peak daily head on top of the barrier layer (i.e. geosynthetic clay liner (GCL) or high density polyethylene (HDPE) geomembrane) for any of the runs made was 0.079 inches associated with a peak daily precipitation of 6.16 inches. This is well below the SCDHEC limit of 12 inches. 


\section{TABLE OF CONTENTS}

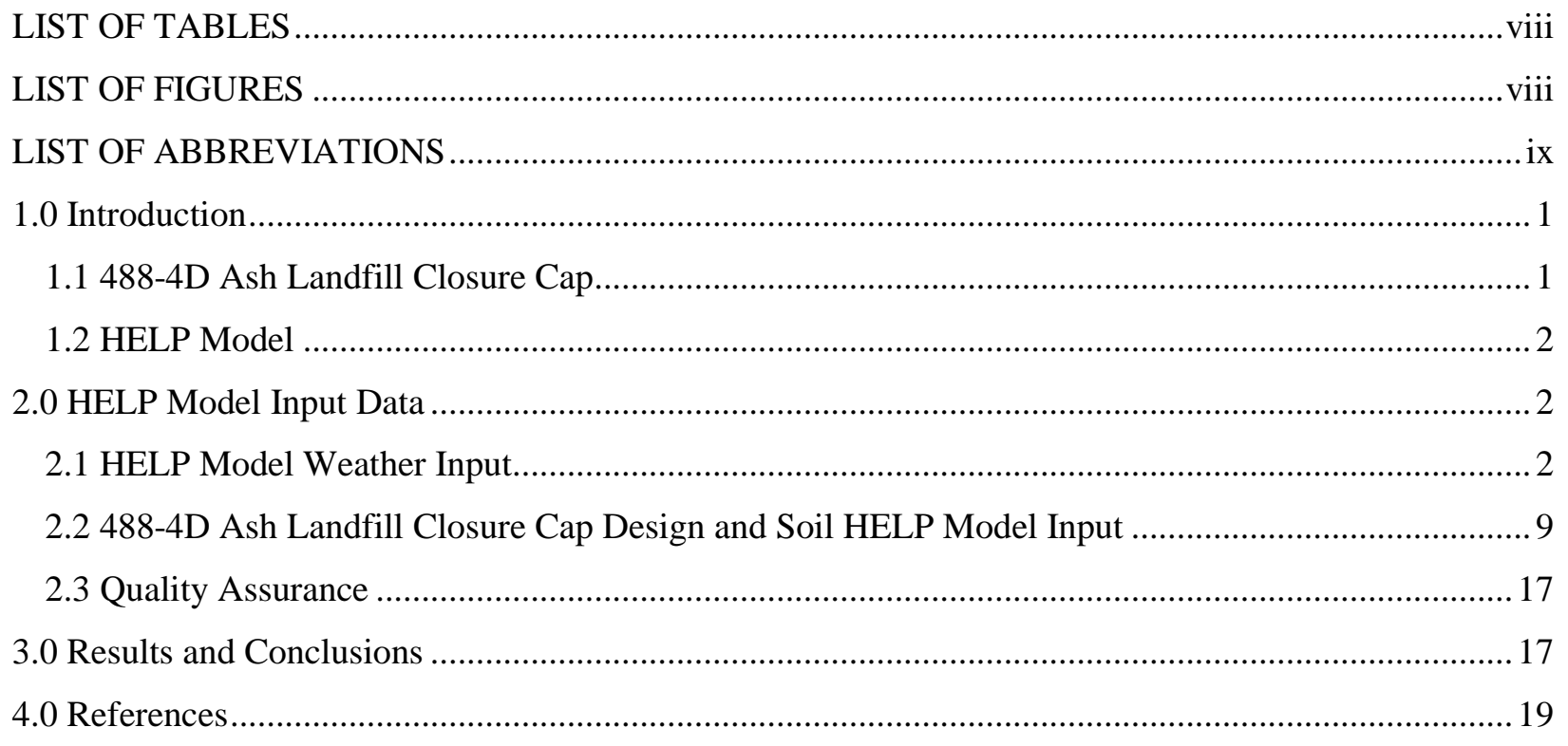




\section{LIST OF TABLES}

Table 2-1. SRS Specific Precipitation Data ……................................................................................... 4

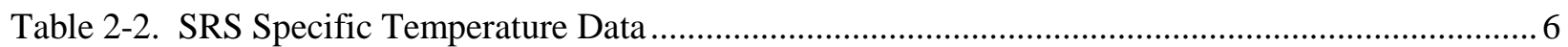

Table 2-3. HELP Model Input Data for D-Area Ash Landfill Base Case Closure Cap with GCL Barrier

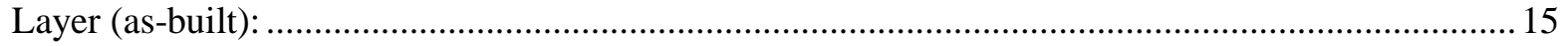

Table 2-4. HELP Model Input Data for D-Area Ash Landfill Alternative Case Closure Cap with HDPE Geomembrane Barrier Layer (as-built): .......................................................................................... 16

Table 3-1. 488-4D HELP Model Summary Output ................................................................................ 18

\section{LIST OF FIGURES}

Figure 1-1. 488-4D Closure Cap Profile ............................................................................................... 1

Figure 2-1. Nominal Surface and Drain Slopes and Lengths (C-CG-D-00010) ..................................... 10

Figure 2-2. Corner Surface and Drain Slopes and Lengths (C-CG-D-00011)....................................... 10 


\section{LIST OF ABBREVIATIONS}

$\begin{array}{ll}\text { ACP } & \text { Area Completion Projects } \\ \text { CLM } & \text { Central Climatology site } \\ \text { ET } & \text { evapotranspiration } \\ \text { GCL } & \text { geosynthetic clay liner } \\ \text { GDL } & \text { geosynthetic drainage layer } \\ \text { HDPE } & \text { high density polyethylene } \\ \text { HELP } & \text { Hydrologic Evaluation of Landfill Performance } \\ \text { LS } & \text { loamy sand } \\ \text { SC } & \text { clayey sand } \\ \text { SCDHEC } & \text { South Carolina Department of Health and Environmental Control } \\ \text { SCL } & \text { sandy clay loam } \\ \text { SM } & \text { silty sand } \\ \text { SRNL } & \text { Savannah River National Laboratory } \\ \text { SRNS } & \text { Savannah River Nuclear Solutions } \\ \text { SRS } & \text { Savannah River Site } \\ \text { USACE } & \text { United States Army Corps of Engineers } \\ \text { USCS } & \text { Unified Soil Classification System } \\ \text { USDA } & \text { United States Department of Agriculture } \\ \text { USEPA } & \text { U.S. Environmental Protection Agency } \\ \text { WES } & \text { Waterways Experiment Station }\end{array}$


SRNL-STI-2014-00392

Revision 1

Intentionally Blank 


\subsection{Introduction}

The South Carolina Department of Health and Environmental Control (SCDHEC) has requested that Savannah River Nuclear Solutions (SRNS) perform Hydrologic Evaluation of Landfill Performance (HELP) modeling of the planned 488-4D Ash Landfill closure cap to ensure that no more than a 12 inch head will develop on top of the barrier layer (saturated hydraulic conductivity of no more than 1.0E-05 $\mathrm{cm} / \mathrm{s}$ ) in association with a 25-year, 24-hour storm event. In addition SCDHEC has requested that HELP modeling also be performed for an alternative closure cap that utilizes high density polyethylene (HDPE) geomembrane as the barrier layer rather than the current geosynthetic clay liner (GCL).

This report documents performance of the requested HELP modeling as follows:

- $\quad$ Section 1.1 provides a brief overview of the 488-4D closure cap.

- Section 1.2 provides a brief overview of the HELP model.

- Section 2.1 provides an overview of the development of the HELP model weather input.

- Section 2.2 provides an overview of the development of the 488-4D Ash Landfill soil, geomembrane, and closure cap design input.

- Section 3.0 provides the results and conclusions.

\subsection{8-4D Ash Landfill Closure Cap}

Removal of ash from the 488-2D Ash Landfill, placement of the 488-2D ash within the 488-4D Ash Landfill, and construction of a closure cap over the 488-4D Ash Landfill is planned (C-SPP-D-00002). Figure 1-1 provides the planned profile of the 488-4D closure cap (C-CG-D-00017). HELP modeling has been performed based upon this base case profile and for an alternative profile where the GCL has been replaced with a HDPE geomembrane.

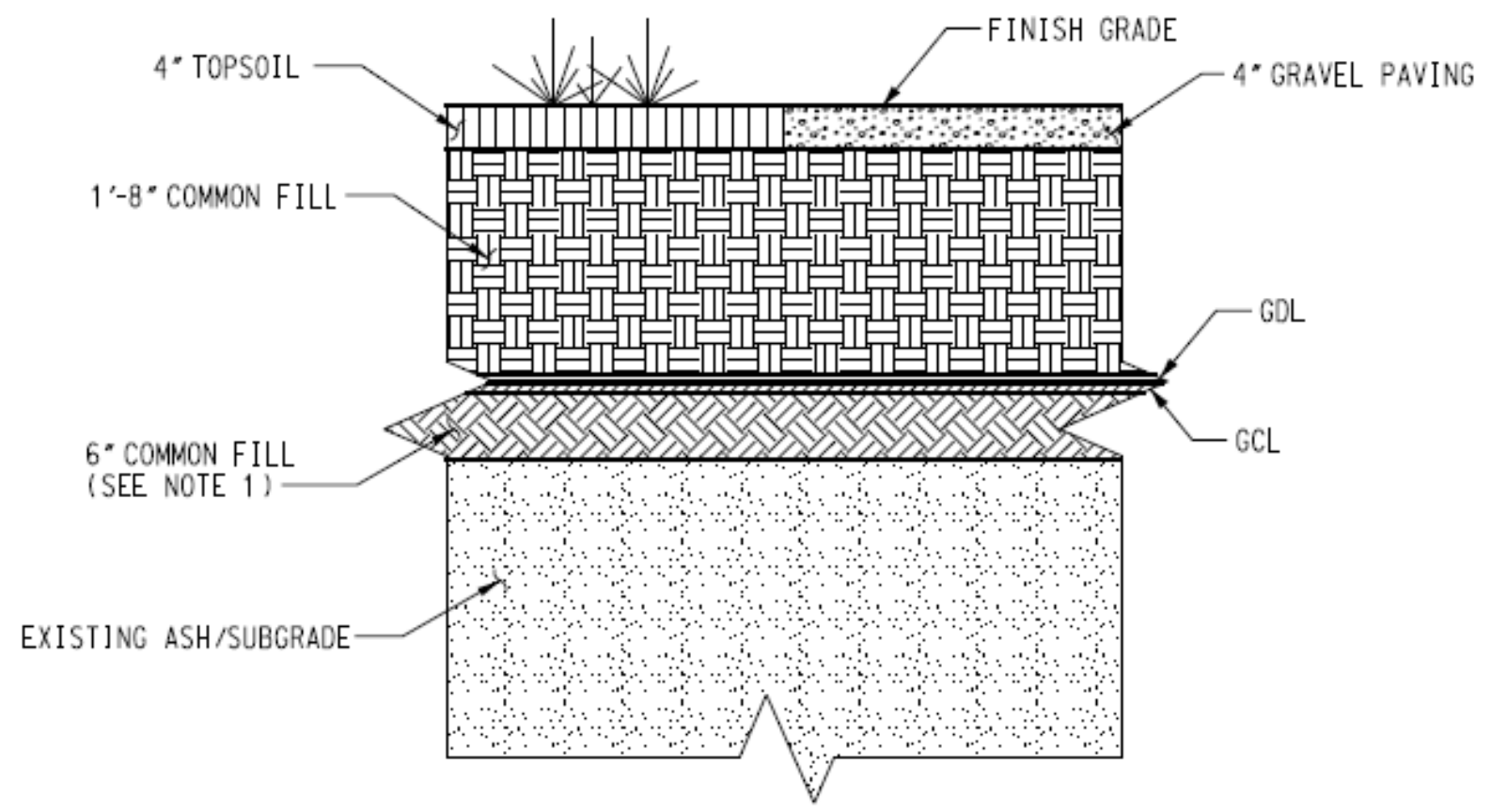

Figure 1-1. 488-4D Closure Cap Profile

(GDL = geosynthetic drainage layer; GCL = geosynthetic clay liner) 


\subsection{HELP Model}

The HELP model is a quasi-two-dimensional water balance model designed to conduct landfill water balance analyses. The model requires the input of weather, soil, and closure cap design data. It provides estimates of runoff, evapotranspiration, lateral drainage, vertical percolation (i.e., infiltration), hydraulic head, and water storage for the evaluation of various landfill designs.

United States Army Corps of Engineers (USACE) personnel at the Waterways Experiment Station (WES) in Vicksburg, Mississippi developed the HELP model, under an interagency agreement (DW21931425) with the U.S. Environmental Protection Agency (USEPA). As such the HELP model is a USEPAsanctioned model for conducting landfill water balance analyses. HELP model version 3.07, issued on November 1, 1997, is the latest version of the model. It is public domain software available from the WES website at:

http://el.erdc.usace.army.mil/products.cfm?Topic=model\&Type=landfill.

USEPA and the USACE have provided the following documentation associated with the HELP model:

- A user’s guide (Schroeder et al. 1994a), which provides instructions for HELP model use.

- Engineering documentation (Schroeder et al. 1994b), which provides information on the source language used to write the code, the hardware necessary to operate the code, data generation methodologies available for use, and the methods of solution.

- Verification test reports comparing the model's drainage layer estimates to the results of largescale physical models (Schroeder et al. 1987a) and comparing the model's water balance estimates to "field data from a total of 20 landfill cells at 7 sites in the United States" (Schroeder et al. 1987b).

\subsection{HELP Model Input Data}

\subsection{HELP Model Weather Input}

Required HELP model weather input includes:

- Precipitation data

- Temperature data

- Solar Radiation data

- Evapotranspiration data

The HELP model has a synthetic weather data option for the creation of precipitation, temperature, and solar radiation data. The option allows the generation of synthetic weather data based upon city specific data stored within the HELP model modified by the user's site specific data. Therefore synthetic daily precipitation and temperature data over 100 years has been generated based upon the HELP synthetic weather data option for Augusta, GA, that has been modified with the SRS specific average monthly precipitation and temperature data, respectively. SRS collects meteorological data from a network of weather stations. The primary SRS precipitation data has been collected from the Savannah River National Laboratory (SRNL) (773-A) weather station between 1952 and 1995 and from the Central Climatology site (CLM) since 1995. The primary SRS temperature data has been collected from the SRNL weather station between 1968 and 1995 and from the CLM since 1995. This data (Tables 2-1 and 2-2, respectively) has been extracted from the Atmospheric Technologies Center web page 
(http://shweather.srs.gov/Weather/) and has been used to generate the HELP model precipitation and temperature input data. The synthetic weather data option for Augusta, GA, has also been utilized to generate the solar radiation input data, and the default Augusta, GA values for evapotranspiration data have been used to generate the evapotranspiration input data. The evaporative zone depth has been set to 24 inches based upon the thickness of soil material over the GDL and the acceptable range of evaporative zone depth for Augusta, GA, of 10 to 40 inches. The maximum leaf area index has been set at 3.5, which represents a good stand of grass, based upon the sodding and permanent seeding requirements of Specification C-SPP-D-00002. The following are the weather input files which have been generated:

- $\quad$ Precipitation data: DALPREC.D4

- Temperature data: DALTEMP.D7

- Solar Radiation data: DALSOLR.D13

- Evapotranspiration data: DALEVAP.D11 
Table 2-1. SRS Specific Precipitation Data

\begin{tabular}{|c|c|c|c|c|c|c|c|c|c|c|c|c|}
\hline Year & $\begin{array}{c}\text { Jan } \\
\text { Precip. } \\
\text { (inches) }\end{array}$ & $\begin{array}{c}\text { Feb } \\
\text { Precip. } \\
\text { (inches) }\end{array}$ & $\begin{array}{l}\text { March } \\
\text { Precip. } \\
\text { (inches) }\end{array}$ & $\begin{array}{c}\text { April } \\
\text { Precip. } \\
\text { (inches) }\end{array}$ & $\begin{array}{c}\text { May } \\
\text { Precip. } \\
\text { (inches) }\end{array}$ & $\begin{array}{c}\text { June } \\
\text { Precip. } \\
\text { (inches) }\end{array}$ & $\begin{array}{c}\text { July } \\
\text { Precip. } \\
\text { (inches) }\end{array}$ & $\begin{array}{c}\text { Aug } \\
\text { Precip. } \\
\text { (inches) }\end{array}$ & $\begin{array}{c}\text { Sept } \\
\text { Precip. } \\
\text { (inches) }\end{array}$ & $\begin{array}{c}\text { Oct } \\
\text { Precip. } \\
\text { (inches) }\end{array}$ & $\begin{array}{c}\text { Nov } \\
\text { Precip. } \\
\text { (inches) }\end{array}$ & $\begin{array}{c}\text { Dec } \\
\text { Precip. } \\
\text { (inches) }\end{array}$ \\
\hline 1952 & 2.07 & 3.23 & 6.55 & 3.12 & 5.56 & 5.67 & 2.82 & 5.98 & 3.34 & 1.36 & 2.86 & 3.99 \\
\hline 1953 & 2.69 & 5.48 & 3.83 & 2.96 & 4.42 & 5.38 & 3.63 & 3.61 & 8.53 & 0.11 & 1.04 & 7.51 \\
\hline 1954 & 1.26 & 1.64 & 2.95 & 2.5 & 2.89 & 2.91 & 2.03 & 4.1 & 1.43 & 1.29 & 2.94 & 2.88 \\
\hline 1955 & 4.75 & 2.62 & 2.21 & 5.57 & 4.53 & 3.31 & 3.94 & 5.07 & 3.42 & 1.32 & 2.93 & 0.46 \\
\hline 1956 & 1.67 & 7.94 & 4.84 & 3.21 & 3.07 & 2.34 & 4.34 & 3.18 & 4.56 & 1.83 & 0.93 & 2.05 \\
\hline 1957 & 2.05 & 1.58 & 4.29 & 2.75 & 8.02 & 4.17 & 3.51 & 2.41 & 5.04 & 6.12 & 6.46 & 2.24 \\
\hline 1958 & 4.01 & 4.38 & 4.96 & 5.63 & 2.07 & 2.5 & 5.32 & 2.76 & 1.12 & 0.96 & 0.21 & 4.42 \\
\hline 1959 & 3.54 & 6.06 & 6.44 & 2.03 & 3.81 & 4.06 & 5.8 & 2.93 & 8.71 & 10.86 & 1.97 & 3.54 \\
\hline 1960 & 6.91 & 5.81 & 5.76 & 5.07 & 1.96 & 3.66 & 5.27 & 2.81 & 4.84 & 0.97 & 0.83 & 2.93 \\
\hline 1961 & 3.59 & 5.76 & 7.23 & 8.2 & 3.88 & 3.01 & 3.09 & 7.15 & 1 & 0.07 & 1.83 & 6.6 \\
\hline 1962 & 4.64 & 5.14 & 6.52 & 4.03 & 3.5 & 4.41 & 2.56 & 3.43 & 5.55 & 2.27 & 3.5 & 2.2 \\
\hline 1963 & 5.96 & 3.64 & 3.34 & 3.7 & 2.98 & 8.42 & 3.18 & 1.04 & 5.37 & 0 & 3.68 & 4.47 \\
\hline 1964 & 7.79 & 6 & 5.79 & 5.94 & 3.62 & 4.5 & 10.42 & 12.34 & 5.43 & 6.53 & 0.6 & 4.1 \\
\hline 1965 & 1.83 & 6.19 & 10.18 & 2.81 & 1.63 & 5.14 & 9.57 & 1.29 & 2.36 & 2.95 & 1.99 & 1.69 \\
\hline 1966 & 7.81 & 6.22 & 4.3 & 2.93 & 5.28 & 4.81 & 3.52 & 5.84 & 3.98 & 1.51 & 1.37 & 3.85 \\
\hline 1967 & 3.91 & 4.43 & 7.54 & 2.6 & 5.94 & 4.06 & 7.23 & 8.48 & 0.99 & 0.31 & 2.81 & 3.37 \\
\hline 1968 & 4.56 & 0.97 & 1.58 & 2.23 & 4.24 & 5.28 & 3.58 & 8.05 & 5.06 & 3.33 & 4.14 & 2.93 \\
\hline 1969 & 2.2 & 2.47 & 3.42 & 4.71 & 2.57 & 4.26 & 1.94 & 4.38 & 4.05 & 2 & 0.4 & 4.42 \\
\hline 1970 & 3.12 & 2.75 & 7.9 & 1.28 & 4.01 & 4.68 & 4.69 & 3.78 & 2.75 & 4.02 & 1.5 & 5.62 \\
\hline 1971 & 5.01 & 3.8 & 9.71 & 2.57 & 3.62 & 4.81 & 13.71 & 9.98 & 4.74 & 5.27 & 2.16 & 2.79 \\
\hline 1972 & 7.81 & 3.71 & 2.68 & 0.6 & 4.1 & 5.64 & 1.92 & 8.19 & 1.52 & 1.03 & 2.92 & 4.26 \\
\hline 1973 & 5.5 & 4.47 & 6.67 & 4.55 & 4.91 & 12.97 & 6.86 & 3.9 & 4.38 & 1.72 & 0.98 & 3.99 \\
\hline 1974 & 2.42 & 6.66 & 3.03 & 3.05 & 3.35 & 2.8 & 4.44 & 6.77 & 3.32 & 0.09 & 1.99 & 4.11 \\
\hline 1975 & 4.98 & 6.64 & 5.92 & 4.42 & 5.15 & 3.83 & 8.55 & 3.83 & 5.18 & 1.74 & 3.41 & 2.03 \\
\hline 1976 & 4.18 & 1.08 & 3.83 & 2.5 & 10.9 & 4.35 & 1.95 & 1.64 & 5.48 & 4.92 & 4.19 & 5.08 \\
\hline 1977 & 3.72 & 1.62 & 6.86 & 1.27 & 1.79 & 2.47 & 3.42 & 7.3 & 5.5 & 4.27 & 1.63 & 3.86 \\
\hline
\end{tabular}




\begin{tabular}{|c|c|c|c|c|c|c|c|c|c|c|c|c|}
\hline Year & $\begin{array}{c}\text { Jan } \\
\text { Precip. } \\
\text { (inches) }\end{array}$ & $\begin{array}{c}\text { Feb } \\
\text { Precip. } \\
\text { (inches) }\end{array}$ & $\begin{array}{l}\text { March } \\
\text { Precip. } \\
\text { (inches) }\end{array}$ & $\begin{array}{c}\text { April } \\
\text { Precip. } \\
\text { (inches) }\end{array}$ & $\begin{array}{c}\text { May } \\
\text { Precip. } \\
\text { (inches) }\end{array}$ & $\begin{array}{c}\text { June } \\
\text { Precip. } \\
\text { (inches) }\end{array}$ & $\begin{array}{c}\text { July } \\
\text { Precip. } \\
\text { (inches) }\end{array}$ & $\begin{array}{c}\text { Aug } \\
\text { Precip. } \\
\text { (inches) }\end{array}$ & $\begin{array}{c}\text { Sept } \\
\text { Precip. } \\
\text { (inches) }\end{array}$ & $\begin{array}{c}\text { Oct } \\
\text { Precip. } \\
\text { (inches) }\end{array}$ & $\begin{array}{c}\text { Nov } \\
\text { Precip. } \\
\text { (inches) }\end{array}$ & $\begin{array}{c}\text { Dec } \\
\text { Precip. } \\
\text { (inches) }\end{array}$ \\
\hline 1978 & 10.02 & 1.31 & 3.06 & 3.53 & 3.64 & 3.42 & 4.11 & 5.1 & 4.06 & 0.06 & 3.54 & 1.87 \\
\hline 1979 & 3.59 & 7.74 & 3.09 & 6.49 & 8.94 & 1.54 & 7.85 & 2.12 & 6.13 & 1.35 & 3.95 & 2.17 \\
\hline 1980 & 5.12 & 3.48 & 10.96 & 1.69 & 3.49 & 2.99 & 0.9 & 2.03 & 5.86 & 2.14 & 2.5 & 1.91 \\
\hline 1981 & 0.89 & 5.02 & 4.72 & 2.07 & 6.9 & 4.29 & 3.96 & 5.79 & 0.54 & 2.81 & 1 & 9.55 \\
\hline 1982 & 3.94 & 4.46 & 2.51 & 5.68 & 2.73 & 4.28 & 11.49 & 5.02 & 4.62 & 3.87 & 2.41 & 4.85 \\
\hline 1983 & 3.75 & 7.22 & 6.62 & 5.77 & 1.67 & 6.57 & 4.85 & 6.32 & 3.56 & 1.92 & 5.39 & 4.15 \\
\hline 1984 & 3.51 & 7.09 & 6.05 & 8 & 9.79 & 2.54 & 7.28 & 5.52 & 0.6 & 0.31 & 0.9 & 1.38 \\
\hline 1985 & 3.01 & 6.92 & 1.31 & 0.84 & 1.7 & 4.62 & 8.1 & 4.38 & 0.49 & 6.34 & 6.36 & 2.48 \\
\hline 1986 & 1.46 & 3.58 & 4.08 & 1.45 & 3.84 & 3.03 & 2.96 & 10.9 & 1.54 & 4.19 & 5.82 & 5.83 \\
\hline 1987 & 7.39 & 7.55 & 4.97 & 0.7 & 3.57 & 5.64 & 4.87 & 4.93 & 3.56 & 0.29 & 2.74 & 1.42 \\
\hline 1988 & 4.15 & 3.19 & 2.91 & 4.78 & 2.85 & 7.12 & 1.78 & 6.8 & 4.4 & 3.39 & 2.17 & 2.91 \\
\hline 1989 & 1.42 & 3.59 & 5.52 & 4.89 & 2.6 & 6.67 & 11.46 & 3.27 & 4.87 & 3.36 & 3 & 4.41 \\
\hline 1990 & 3.07 & 2.38 & 2.37 & 1.21 & 2.95 & 0.89 & 7.31 & 8.07 & 0.62 & 19.62 & 1.41 & 1.57 \\
\hline 1991 & 7.03 & 1.84 & 7.89 & 4.73 & 3.06 & 2.17 & 7.89 & 9.26 & 4.4 & 0.99 & 1.55 & 3.32 \\
\hline 1992 & 4.45 & 3.89 & 2.98 & 2.4 & 1.34 & 6.27 & 3.69 & 4.83 & 6.38 & 3.11 & 7.78 & 2.86 \\
\hline 1993 & 7.45 & 3.62 & 8.37 & 1.74 & 1.43 & 3.27 & 3.12 & 2.23 & 7.29 & 0.99 & 1.87 & 1.81 \\
\hline 1994 & 4.8 & 3.91 & 6.42 & 1.05 & 1.45 & 5.08 & 7.47 & 3.47 & 0.99 & 10.01 & 3.05 & 4.62 \\
\hline 1995 & 6.96 & 7.97 & 0.92 & 1.28 & 1.77 & 8.15 & 5.71 & 6.92 & 5.75 & 2.64 & 2.38 & 4.47 \\
\hline 1996 & 3.65 & 2.43 & 6.64 & 2.4 & 2.96 & 3.04 & 5.57 & 6.91 & 3.67 & 2.16 & 2.32 & 3.2 \\
\hline 1997 & 4.2 & 5.45 & 2.69 & 4.38 & 2.38 & 6.9 & 7.09 & 2.01 & 4.89 & 4.08 & 5.51 & 9.09 \\
\hline 1998 & 7.73 & 8.9 & 6.69 & 7.35 & 4.05 & 4.65 & 5.27 & 2.88 & 4.81 & 0.78 & 0.82 & 1.8 \\
\hline 1999 & 5.31 & 2.29 & 3.44 & 1.95 & 1.26 & 7.52 & 4.91 & 3.14 & 4.46 & 2.57 & 1.5 & 1.21 \\
\hline 2000 & 5.77 & 0.73 & 3.95 & 1.34 & 1.36 & 4.74 & 2.47 & 4.49 & 7.7 & 0.02 & 3.5 & 1.53 \\
\hline 2001 & 3.11 & 2.68 & 7.21 & 1.28 & 3.85 & 6.49 & 4.79 & 3.55 & 3.33 & 0.5 & 1.03 & 0.54 \\
\hline 2002 & 2.85 & 2.13 & 3.86 & 2.58 & 1.69 & 2.3 & 5.95 & 5.47 & 3.45 & 3.19 & 4 & 3.58 \\
\hline 2003 & 1.73 & 5 & 7.09 & 8.43 & 5.57 & 10.99 & 8.91 & 4.59 & 2.7 & 3.03 & 1.21 & 1.93 \\
\hline 2004 & 2.85 & 6.71 & 0.81 & 1.34 & 3.45 & 6.41 & 1.23 & 2.96 & 10.26 & 1.02 & 3.17 & 2.69 \\
\hline
\end{tabular}




\begin{tabular}{|c|c|c|c|c|c|c|c|c|c|c|c|c|}
\hline Year & $\begin{array}{c}\text { Jan } \\
\text { Precip. } \\
\text { (inches) } \\
\end{array}$ & $\begin{array}{c}\text { Feb } \\
\text { Precip. } \\
\text { (inches) }\end{array}$ & $\begin{array}{l}\text { March } \\
\text { Precip. } \\
\text { (inches) }\end{array}$ & $\begin{array}{c}\text { April } \\
\text { Precip. } \\
\text { (inches) }\end{array}$ & $\begin{array}{c}\text { May } \\
\text { Precip. } \\
\text { (inches) }\end{array}$ & $\begin{array}{c}\text { June } \\
\text { Precip. } \\
\text { (inches) } \\
\end{array}$ & $\begin{array}{c}\text { July } \\
\text { Precip. } \\
\text { (inches) }\end{array}$ & $\begin{array}{c}\text { Aug } \\
\text { Precip. } \\
\text { (inches) }\end{array}$ & $\begin{array}{c}\text { Sept } \\
\text { Precip. } \\
\text { (inches) }\end{array}$ & $\begin{array}{c}\text { Oct } \\
\text { Precip. } \\
\text { (inches) }\end{array}$ & $\begin{array}{c}\text { Nov } \\
\text { Precip. } \\
\text { (inches) }\end{array}$ & $\begin{array}{c}\text { Dec } \\
\text { Precip. } \\
\text { (inches) }\end{array}$ \\
\hline 2005 & 2.14 & 3.89 & 6.09 & 1.69 & 2.87 & 8.23 & 5.81 & 4.08 & 0.19 & 3.6 & 2.67 & 6.16 \\
\hline 2006 & 3.38 & 2.9 & 1.76 & 2.41 & 1.83 & 6.89 & 5.22 & 2.19 & 2.5 & 1.66 & 2.98 & 4.56 \\
\hline 2007 & 3.27 & 3.6 & 1.98 & 2.95 & 1.23 & 4.83 & 4.57 & 2.66 & 0.97 & 1.35 & 0.55 & 8.79 \\
\hline 2008 & 3.72 & 5.36 & 3.04 & 2.39 & 1.82 & 1.37 & 5.44 & 5.4 & 0.94 & 4.12 & 5.14 & 2.87 \\
\hline 2009 & 1.98 & 1.68 & 3.65 & 4.6 & 5.2 & 2.73 & 2.56 & 3.13 & 3.73 & 3 & 5.45 & 10.24 \\
\hline 2010 & 4.83 & 2.37 & 3.03 & 1.51 & 2.56 & 5.65 & 2.74 & 5.22 & 2.86 & 0.31 & 1.32 & 1.34 \\
\hline 2011 & 2.24 & 4.92 & 5.5 & 1.83 & 1.1 & 1.47 & 4 & 2.98 & 4.34 & 2.07 & 1.13 & 1.66 \\
\hline 2012 & 1.8 & 1.5 & 3.57 & 1.78 & 8.93 & 2.37 & 5.95 & 6.36 & 2.57 & 0.29 & 1.29 & 4.8 \\
\hline 2013 & 0.77 & 10.11 & 3.25 & 5.04 & 2.36 & 9.64 & 12.92 & 5.64 & 1.14 & 1.44 & 1.72 & 4.6 \\
\hline Ave & 4.05 & 4.32 & 4.80 & 3.29 & 3.65 & 4.75 & 5.28 & 4.85 & 3.84 & 2.73 & 2.62 & 3.67 \\
\hline
\end{tabular}

Table 2-2. SRS Specific Temperature Data

\begin{tabular}{|c|c|c|c|c|c|c|c|c|c|c|c|c|}
\hline Year & $\begin{array}{c}\text { Jan } \\
\text { Temp. } \\
\left({ }^{\circ} \mathbf{F}\right) \\
\end{array}$ & $\begin{array}{c}\text { Feb } \\
\text { Temp. } \\
\left({ }^{\circ} \mathbf{F}\right) \\
\end{array}$ & $\begin{array}{c}\text { March } \\
\text { Temp. } \\
\left({ }^{0} \mathbf{F}\right) \\
\end{array}$ & $\begin{array}{c}\text { April } \\
\text { Temp. } \\
\left({ }^{0} \mathbf{F}\right) \\
\end{array}$ & $\begin{array}{c}\text { May } \\
\text { Temp. } \\
\left({ }^{\mathbf{0}} \mathbf{F}\right)\end{array}$ & $\begin{array}{c}\text { June } \\
\text { Temp. } \\
\left({ }^{\mathbf{0}} \mathbf{F}\right) \\
\end{array}$ & $\begin{array}{c}\text { July } \\
\text { Temp. } \\
\left({ }^{0} \mathrm{~F}\right) \\
\end{array}$ & $\begin{array}{c}\text { Aug } \\
\text { Temp. } \\
\left({ }^{\circ} \mathbf{F}\right) \\
\end{array}$ & $\begin{array}{c}\text { Sept } \\
\text { Temp. } \\
\left({ }^{\circ} \mathbf{F}\right) \\
\end{array}$ & $\begin{array}{c}\text { Oct } \\
\text { Temp. } \\
\left({ }^{0} \mathbf{F}\right) \\
\end{array}$ & $\begin{array}{c}\text { Nov } \\
\text { Temp. } \\
\left({ }^{\mathbf{0}} \mathbf{F}\right) \\
\end{array}$ & $\begin{array}{c}\text { Dec } \\
\text { Temp. } \\
\left({ }^{0} \mathrm{~F}\right) \\
\end{array}$ \\
\hline 1968 & 43.5 & 43.4 & 57.1 & 66.5 & 71.3 & 80 & 83.1 & 82.8 & 77 & 67 & 55.4 & 45.9 \\
\hline 1969 & 46.5 & 46.6 & 51.5 & 64.5 & 70.5 & 80.3 & 83.3 & 77.6 & 72.8 & 66.1 & 52.1 & 45.4 \\
\hline 1970 & 39 & 47.2 & 55.9 & 66.8 & 74.2 & 79 & 81.1 & 80.8 & 78.6 & 67 & 51.6 & 49.3 \\
\hline 1971 & 44.6 & 46.4 & 49.5 & 63.4 & 70.7 & 81.3 & 80.7 & 80.4 & 75.2 & 70.2 & 55.5 & 56.9 \\
\hline 1972 & 51.7 & 45.6 & 57.6 & 67.4 & 72.4 & 75.3 & 79.7 & 80.6 & 77.2 & 64.8 & 54.4 & 53.2 \\
\hline 1973 & 46.1 & 45.9 & 60.7 & 61.9 & 70.5 & 77.7 & 79.1 & 74.5 & 70.5 & 62.4 & 59 & 50.3 \\
\hline 1974 & 59.6 & 50.8 & 62.2 & 66.2 & 75.3 & 77.5 & 81.5 & 80.9 & 75.3 & 64.5 & 56.6 & 49 \\
\hline 1975 & 51.4 & 53.2 & 55.8 & 63.9 & 75.6 & 79.1 & 79.7 & 82.4 & 75.7 & 68.7 & 59.3 & 48.5 \\
\hline 1976 & 44.2 & 55.7 & 61.5 & 64.8 & 68.9 & 75.6 & 80.4 & 78 & 73.1 & 60.1 & 48.7 & 44.8 \\
\hline 1977 & 35.3 & 47.1 & 60 & 66.9 & 73.3 & 80.6 & 83.6 & 80.6 & 77.9 & 62.1 & 58.2 & 46.7 \\
\hline 1978 & 39.3 & 41.3 & 54.2 & 65.7 & 70.9 & 79.7 & 82.1 & 81.2 & 77.1 & 65.6 & 60.7 & 49.6 \\
\hline
\end{tabular}




\begin{tabular}{|c|c|c|c|c|c|c|c|c|c|c|c|c|}
\hline Year & $\begin{array}{c}\text { Jan } \\
\text { Temp. } \\
\left({ }^{\circ} \mathrm{F}\right) \\
\end{array}$ & $\begin{array}{c}\text { Feb } \\
\text { Temp. } \\
\left({ }^{0} \mathbf{F}\right) \\
\end{array}$ & $\begin{array}{c}\text { March } \\
\text { Temp. } \\
\left({ }^{\mathbf{0}} \mathbf{F}\right) \\
\end{array}$ & $\begin{array}{c}\text { April } \\
\text { Temp. } \\
\left({ }^{0} \mathrm{~F}\right) \\
\end{array}$ & $\begin{array}{c}\text { May } \\
\text { Temp. } \\
\left({ }^{\circ} \mathrm{F}\right) \\
\end{array}$ & $\begin{array}{c}\text { June } \\
\text { Temp. } \\
\left({ }^{0} \mathbf{F}\right) \\
\end{array}$ & $\begin{array}{c}\text { July } \\
\text { Temp. } \\
\left({ }^{\mathbf{0}} \mathbf{F}\right) \\
\end{array}$ & $\begin{array}{c}\text { Aug } \\
\text { Temp. } \\
\left({ }^{0} \mathrm{~F}\right) \\
\end{array}$ & $\begin{array}{c}\text { Sept } \\
\text { Temp. } \\
\left({ }^{0} \mathrm{~F}\right) \\
\end{array}$ & $\begin{array}{c}\text { Oct } \\
\text { Temp. } \\
\left({ }^{0} \mathrm{~F}\right) \\
\end{array}$ & $\begin{array}{c}\text { Nov } \\
\text { Temp. } \\
\left({ }^{0} \mathbf{F}\right) \\
\end{array}$ & $\begin{array}{c}\text { Dec } \\
\text { Temp. } \\
\left({ }^{0} \mathbf{F}\right) \\
\end{array}$ \\
\hline 1979 & 42.1 & 44.6 & 57.5 & 64.5 & 71.3 & 75.1 & 79.6 & 80.5 & 73.4 & 64.8 & 57.4 & 47.4 \\
\hline 1980 & 45.9 & 44.3 & 52.6 & 63.5 & 71.2 & 78.3 & 83.8 & 82.5 & 79.2 & 62.7 & 52.8 & 46 \\
\hline 1981 & 40.4 & 48.5 & 53 & 67 & 68.6 & 81.3 & 81.3 & 76.3 & 74 & 62.1 & 54.4 & 43.2 \\
\hline 1982 & 43 & 50 & 58.9 & 62.4 & 75.7 & 78.8 & 80.9 & 80.1 & 75 & 66.2 & 58.7 & 54.8 \\
\hline 1983 & 43.3 & 48 & 55.3 & 59.4 & 66.8 & 76.7 & 84.3 & 83.9 & 74.8 & 67.2 & 56.4 & 45.8 \\
\hline 1984 & 45 & 51.7 & 56.5 & 62.6 & 71.9 & 80.1 & 80.1 & 80.8 & 74 & 73.4 & 53.4 & 56.9 \\
\hline 1985 & 42.9 & 49.5 & 60.2 & 67.5 & 74.5 & 80.8 & 81.1 & 79.7 & 75.7 & 70.8 & 65.5 & 45.4 \\
\hline 1986 & 45.4 & 54.6 & 57.9 & 66.4 & 74.4 & 82.7 & 86.9 & 80.1 & 78.4 & 67.1 & 61.3 & 49.3 \\
\hline 1987 & 46.2 & 48.6 & 56.5 & 62.3 & 74.5 & 79.9 & 82.8 & 83.8 & 76.6 & 60.7 & 59.1 & 52.9 \\
\hline 1988 & 42.3 & 47.8 & 56.8 & 64.2 & 70.4 & 76.8 & 81.6 & 81.4 & 75.4 & 61.2 & 58 & 49.1 \\
\hline 1989 & 52.2 & 52 & 58.3 & 64.2 & 70.6 & 79.8 & 81.4 & 80.9 & 75.3 & 67.3 & 52.4 & 44.2 \\
\hline 1990 & 54.9 & 57.5 & 60 & 64 & 72.9 & 80.5 & 83.7 & 83.8 & 79 & 69.4 & 59.9 & 54.6 \\
\hline 1991 & 47.9 & 54.1 & 60.3 & 69.2 & 76.9 & 79.5 & 83.6 & 81.2 & 77.4 & 68.1 & 55.4 & 54 \\
\hline 1992 & 49.5 & 54.1 & 57.2 & 65 & 71.2 & 78.9 & 83.7 & 80.7 & 76.9 & 65 & 57.1 & 48 \\
\hline 1993 & 51.7 & 47.8 & 53.2 & 58.9 & 69.7 & 78.2 & 83.6 & 80 & 75.2 & 62.8 & 55.2 & 43.6 \\
\hline 1994 & 41.5 & 50.1 & 60.2 & 68 & 71.2 & 82.3 & 81.8 & 81.2 & 77.4 & 67.2 & 62.3 & 53.3 \\
\hline 1995 & 45.5 & 49.9 & 58.6 & 65.9 & 73.5 & 75 & 79.9 & 79 & 71.8 & 65.9 & 50.8 & 43.8 \\
\hline 1996 & 44.6 & 50.1 & 50.6 & 61.6 & 72.9 & 76.5 & 79.3 & 76 & 72.7 & 62.1 & 51.6 & 48.8 \\
\hline 1997 & 48.2 & 52.9 & 63.3 & 61.2 & 68.5 & 74 & 80.2 & 79 & 75 & 64.1 & 51.6 & 47 \\
\hline 1998 & 49.7 & 51.1 & 53.6 & 62.7 & 74.6 & 82.1 & 82.6 & 80.3 & 75.8 & 66.9 & 60.5 & 53.6 \\
\hline 1999 & 51.9 & 51.6 & 53.4 & 67.2 & 69.7 & 76.6 & 80.7 & 82.9 & 73.8 & 64.3 & 58.1 & 48.6 \\
\hline 2000 & 44.4 & 50.2 & 58.5 & 60.7 & 75.1 & 78 & 79.9 & 77.6 & 71.7 & 62.5 & 53.1 & 38.2 \\
\hline 2001 & 43.8 & 52.4 & 53 & 63.9 & 71.3 & 75.3 & 77.7 & 78.8 & 71.2 & 62.2 & 60 & 52.4 \\
\hline 2002 & 47.3 & 48 & 57.6 & 68.1 & 70.2 & 77.5 & 80.5 & 78.4 & 75.4 & 66.7 & 51.7 & 44.5 \\
\hline 2003 & 42 & 47.5 & 57.6 & 61.6 & 70.6 & 75.2 & 77.3 & 77.7 & 71.9 & 63.7 & 58.2 & 42.9 \\
\hline 2004 & 43.7 & 45.2 & 58.5 & 63.4 & 74 & 77.7 & 80.1 & 77.3 & 73.2 & 66.2 & 56.1 & 45.8 \\
\hline 2005 & 47.9 & 49 & 53.1 & 60.9 & 68 & 75.4 & 79.4 & 78.8 & 77 & 64.7 & 56.1 & 44.3 \\
\hline
\end{tabular}




\begin{tabular}{|c|c|c|c|c|c|c|c|c|c|c|c|c|}
\hline Year & $\begin{array}{c}\text { Jan } \\
\text { Temp. } \\
\left({ }^{0} \mathbf{F}\right) \\
\end{array}$ & $\begin{array}{c}\text { Feb } \\
\text { Temp. } \\
\left({ }^{0} \mathbf{F}\right) \\
\end{array}$ & $\begin{array}{c}\text { March } \\
\text { Temp. } \\
\left({ }^{0} \mathbf{F}\right)\end{array}$ & $\begin{array}{c}\text { April } \\
\text { Temp. } \\
\left({ }^{0} \mathbf{F}\right)\end{array}$ & $\begin{array}{c}\text { May } \\
\text { Temp. } \\
\left({ }^{0} \mathbf{F}\right)\end{array}$ & $\begin{array}{c}\text { June } \\
\text { Temp. } \\
\left({ }^{0} \mathbf{F}\right)\end{array}$ & $\begin{array}{c}\text { July } \\
\text { Temp. } \\
\left({ }^{0} \mathbf{F}\right)\end{array}$ & $\begin{array}{c}\text { Aug } \\
\text { Temp. } \\
\left({ }^{0} \mathbf{F}\right)\end{array}$ & $\begin{array}{c}\text { Sept } \\
\text { Temp. } \\
\left({ }^{0} \mathrm{~F}\right) \\
\end{array}$ & $\begin{array}{c}\text { Oct } \\
\text { Temp. } \\
\left({ }^{0} \mathrm{~F}\right) \\
\end{array}$ & $\begin{array}{l}\text { Nov } \\
\text { Temp. } \\
\left({ }^{0} \mathrm{~F}\right)\end{array}$ & $\begin{array}{c}\text { Dec } \\
\text { Temp. } \\
\left({ }^{0} \mathbf{F}\right)\end{array}$ \\
\hline 2006 & 50.8 & 47.3 & 55.3 & 66.3 & 70.1 & 76.2 & 80.3 & 80.5 & 72.9 & 62.4 & 53.6 & 50.6 \\
\hline 2007 & 48.6 & 46.4 & 58.4 & 61.8 & 70.2 & 76.5 & 77.4 & 81.9 & 75.2 & 68.7 & 54 & 52.3 \\
\hline 2008 & 43.8 & 51.1 & 55.3 & 61.8 & 70.2 & 80.1 & 78.7 & 77.9 & 73.7 & 61.1 & 50 & 52.1 \\
\hline 2009 & 44.9 & 47.4 & 55.2 & 62.3 & 70.7 & 79.2 & 78.6 & 78.2 & 74.1 & 62.7 & 54.6 & 45.5 \\
\hline 2010 & 40.8 & 41.4 & 51.9 & 64.6 & 73.7 & 80 & 81 & 80 & 76.2 & 64 & 54 & 39.2 \\
\hline 2011 & 41.3 & 50.9 & 56.8 & 66.8 & 72 & 81.4 & 81.9 & 81.3 & 74.1 & 60.8 & 55.6 & 51 \\
\hline 2012 & 49.6 & 52.5 & 63.7 & 65.7 & 72.6 & 74.8 & 80.6 & 76 & 72.8 & 64.3 & 51.5 & 51.2 \\
\hline 2013 & 52.1 & 46.8 & 49.7 & 62.6 & 68.5 & 76.2 & 76.9 & 76.3 & 73.7 & 64.6 & 52.6 & 50.9 \\
\hline Ave & 46.0 & 49.1 & 56.6 & 64.3 & 71.8 & 78.3 & 81.0 & 79.9 & 75.0 & 65.1 & 55.8 & 48.5 \\
\hline
\end{tabular}




\subsection{8-4D Ash Landfill Closure Cap Design and Soil HELP Model Input}

Required HELP model closure cap design, soil, and geomembrane input include:

- Closure cap design data includes: Surface and drainage layer slopes and slope lengths; landfill area; layer thicknesses; and layer types.

- Soil data includes: saturated hydraulic conductivity, porosity, field capacity, and wilting point.

- Geomembrane data includes: saturated hydraulic conductivity, geomembrane pinhole density, geomembrane installation defects, and geomembrane placement quality.

Development of this data for the 488-4D closure cap is outlined below.

Surface Slope and Slope Length \& Drainage Length and Drain Slope: For the 488-4D Ash Landfill Closure Cap it is assumed that the surface slope and lateral drainage layer drainage length and that the surface slope length and lateral drainage layer drain slope are the same for any one flow path because the cover profile is constant over the closure cap (drawing C-CG-D-00017 Detail 5). The HELP model only allows the input of constant surface slopes, slope lengths, drainage lengths, and drain slopes (i.e. they cannot be varied in any one HELP model run). Additionally the HELP model does not have a provision to link multiple runs relative to runoff. Therefore average surface slopes, slope lengths, drainage lengths, and drain slopes have been utilized for 488-D HELP modeling.

Figures 2-1 and 2-2 provide the nominal and corner surface slopes, slope lengths, drainage lengths, and drain slopes (averages) to be used within the HELP model runs. Summary information is provided below:

- Nominal:

- $\quad$ Slope Length $=227.55 \mathrm{ft}$

- $\quad$ Slope $=14.85 \%$

- Corner:

- $\quad$ Slope Length $=212.65 \mathrm{ft}$

- $\quad$ Slope $=13.8 \%$ 
SRNL-STI-2014-00392

Revision 1

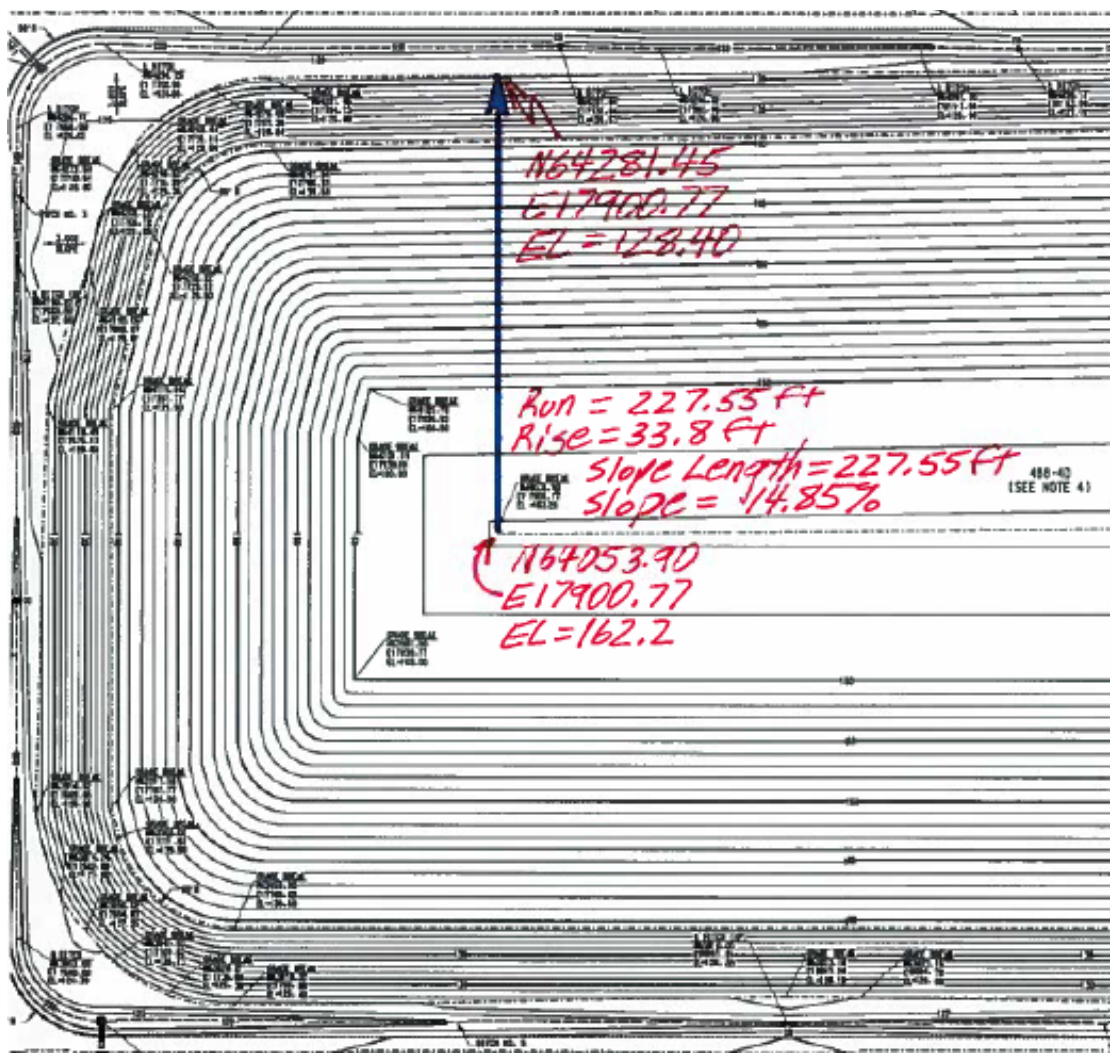

Figure 2-1. Nominal Surface and Drain Slopes and Lengths (C-CG-D-00010)

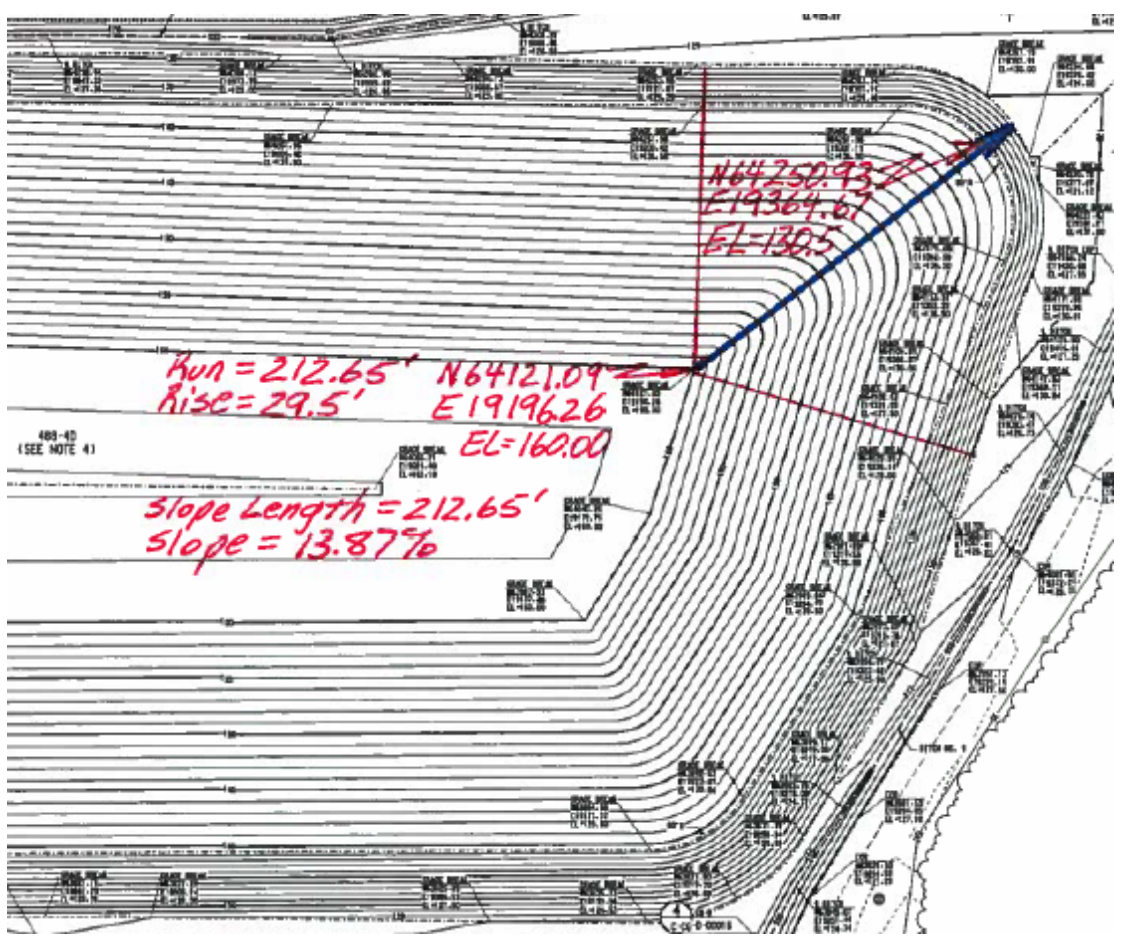

Figure 2-2. Corner Surface and Drain Slopes and Lengths (C-CG-D-00011) 
Topsoil (layer number 1): Properties for the topsoil were developed as follows:

- The topsoil is defined as a vertical percolation layer in HELP.

- Per specification C-SPP-D-00002 the topsoil is to be a "medium textured soil such as loam per ASTM D5268". ASTM D5268 specifies that topsoil have the following particle size range: organic material 2 to 20 ( $<$ No. 10 sieve ashing at $440 \pm 40^{\circ} \mathrm{C}$ ); sand content 20 to 60 ( $<$ No. 10 sieve and retained on No. 200 sieve); and silt and clay content 35 to 70 ( $<$ No. 200 sieve).

- Topsoil thickness is 4 inches per Detail 5 of drawing C-CG-D-00017.

- Yu et al. 1993 provided saturated hydraulic conductivity, total porosity, and water retention (suction head versus saturation) data for two samples of SRS topsoil and Phifer et al. 2007 converted this data into the following required HELP model inputs:

- $\quad$ Saturated hydraulic conductivity $=3.1 \mathrm{E}-03 \mathrm{~cm} / \mathrm{s}$

- $\quad$ Total porosity $(\eta)=0.396$

- $\quad$ Field capacity $=0.109$

- $\quad$ Wilting point $=0.047$

- These topsoil values are similar to the HELP model default soil \#4 that is classified as a silty sand (SM) per the Unified Soil Classification System (USCS) and a loamy sand (LS) per the United States Department of Agriculture (USDA) (Schroeder et al. 1994a, Schroeder et al. 1994b)

Upper and Lower Common Fill (layers number 2 and 5): Properties for the common fill were developed as follows:

- The common fill is defined as a vertical percolation layer in HELP.

- The upper common fill thickness is 20 inches and the lower common fill thickness is 6 inches per Detail 5 of drawing C-CG-D-00017.

- During construction of the low permeability soils cover over the Old Radioactive Burial Grounds, testing of the common fill utilized was conducted for saturated hydraulic conductivity, total porosity, and water retention (suction head versus saturation) data. The following are the required HELP model inputs produced from this data:

- $\quad$ Saturated hydraulic conductivity $=4.4 \mathrm{E}-05 \mathrm{~cm} / \mathrm{s}$

- $\quad$ Total porosity $(\eta)=0.361$

- $\quad$ Field capacity $=0.247$

- $\quad$ Wilting point $=0.202$

- The HELP model defines the field capacity as the volumetric water content $\left(\theta_{\mathrm{v}}\right)$ at a soil suction head $(\Psi)$ of 0.33 bars." (Schroeder et al. 1994a; Schroeder et al. 1994b) 0.33 bars $\approx 337 \mathrm{~cm}-\mathrm{H}_{2} \mathrm{O}$ ( $1 \mathrm{bar} \approx 1,020.7 \mathrm{~cm}-\mathrm{H}_{2} \mathrm{O}$ at $60^{\circ} \mathrm{F}$ ). Field capacity was determined by linear interpolation between two points from the water retention (suction head versus saturation) data.

- The HELP model defines the wilting point as the volumetric water content $\left(\theta_{\mathrm{v}}\right)$ at a soil suction head $(\Psi)$ of 15 bars." (Schroeder et al. 1994a; Schroeder et al. 1994b) 15 bars $\approx 15,310 \mathrm{~cm}-\mathrm{H}_{2} \mathrm{O}$ ( $1 \mathrm{bar} \approx 1,020.7 \mathrm{~cm}-\mathrm{H}_{2} \mathrm{O}$ at $60{ }^{\circ} \mathrm{F}$ ). Site specific data are not available for the determination of the SRS common fill wilting point. Therefore the wilting point from a HELP model default soil that closely resembles the SRS common fill was utilized. Table 2 of Schroeder 1994b provides HELP model moderate and high density default soils. The HELP model default soil \#24, with a wilting point of 0.202 , that is classified as clayey sand (SC) material (USCS) and sandy clay loam (SCL) material (USDA) is considered the closest HELP model default soil to the SRS common fill. Therefore the SRS common fill will be assigned a wilting point of 0.202 for the HELP modeling. 
GDL properties (layer number 3): Properties for the GDL were developed as follows:

- The GDL is defined as a lateral drainage layer in HELP.

- Per specification C-SPP-D-00002 "the GDL shall consist of a HDPE geonet with a nonwoven geotextile fabric heat bonded to both sides ..."

- Agru America Inc. 300 mil Geocomposite (Agru 2013) is considered representative of the GDL to be used.

- Agru America Inc. 300 mil Geocomposite thickness is 0.3 inches (300 mil) (Agru 2013)

- Per Agru 2013 the transmissivity of the double sided (6 oz/yd $\mathrm{yd}^{2}$ geotextile) geocomposite is 9.0E$04 \mathrm{~m}^{2} / \mathrm{s}$. Per Koerner 1990 saturated hydraulic conductivity $\left(K_{\text {sat }}\right)$ equals the transmissivity $(T)$ divided by the thickness $(t)$ :

$$
K_{\text {sat }}=\frac{T}{t}=\frac{9.0 E-04 \mathrm{~m}^{2} / \mathrm{s} \times 10,000 \mathrm{~cm}^{2} / \mathrm{m}^{2}}{0.3 \text { inches } \times 2.54 \mathrm{~cm} / \text { inch }}=11.8 \mathrm{~cm} / \mathrm{s}
$$

- The following are default property values for drainage nets/geonets (Schroeder et al. 1994a, Schroeder et al. 1994b):

- $\quad$ Total porosity $(\eta)=0.850$

- $\quad$ Field capacity $=0.010$

- $\quad$ Wilting point $=0.005$

GCL properties (layer number 4): Properties for the GCL were developed as follows:

- $\quad$ The GCL is defined as a barrier soil liner in HELP.

- Per specification C-SPP-D-00002 the GCL shall consist of a minimum $0.75 \mathrm{lb} / \mathrm{ft}^{2}$ sodium montmorillonite clay (bentonite) evenly distributed between two layers of supporting 6 ounces per square yard nonwoven geotextile.

- $\quad$ CETCO Bentomat ${ }^{\circledR}$ SDN (CETCO 2009) is considered representative of the GCL to be used.

- The typical GCL thickness is 7 to $10 \mathrm{~mm}$ (0.27 to 0.39 inches) (GRI 2013). Will use a thickness of 0.3 inches, as representative.

- The maximum GCL saturated hydraulic conductivity is 5E-09 cm/s per manufacturers' literature (Phifer et al. 2007, CETCO 2009). In addition to the manufacturer's maximum value, the GCL was also run with saturated hydraulic conductivities of 1.0E-08, 1.0E-07, 1.0E-06, and 1.0E-05 $\mathrm{cm} / \mathrm{s}$ (i.e. SCDHEC required saturated hydraulic conductivity for the barrier layer).

- The following are default property values for bentonite/GCLs (Schroeder et al. 1994a, Schroeder et al. 1994b):

- $\quad$ Total porosity $(\eta)=0.750$

- $\quad$ Field capacity $=0.747$

- $\quad$ Wilting point $=0.400$

HDPE geomembrane properties (alternative layer number 4): Properties for the HDPE geomembrane were developed as follows:

- The HDPE geomembrane is defined as a geomembrane liner in HELP.

- The typical thickness of a HDPE geomembrane utilized for closure caps is 60 mils (0.06 inches).

- The default saturated hydraulic conductivity value for HDPE geomembranes (Schroeder et al. 1994a, Schroeder et al. 1994b) is 2.0E-13 cm/s.

- Schroeder et al. 1994a and Schroeder et al. 1994b recommend that "typical geomembranes may have about 0.5 to 1 pinhole per acre from manufacturing defects." Based upon this guidance, the as-installed HDPE geomembrane will be assumed to have 1 pinhole/acre. 
- Schroeder et al. 1994a and Schroeder et al. 1994b recommend an installation defect density of 1 defect per acre for intensively monitored projects and 10 defects per acre or more "when quality assurance is limited to spot checks or when environmental difficulties are encountered during construction”. The as-installed HDPE geomembrane will be assumed to be installed with good quality assurance and will be assumed to have 4 installation defects/acre consistent with the recommendations of Schroeder et al. 1994a and Schroeder et al. 1994b.

- Under the conditions of placement of an HDPE geomembrane directly on top of common fill, Schroeder et al. 1994a and Schroeder et al. 1994b recommend a "good" (HELP model numerical designation 3) geomembrane placement quality designation.

Fly Ash properties (layer number 6): Properties for the fly ash were developed as follows:

- The fly ash is defined as a vertical percolation layer in HELP.

- The maximum thickness of the fly ash layer is approximately 549.6 inches.

- $\quad$ From C-CG-D-00012 the original 488-4D Ash Landfill bottom was $114 \mathrm{ft}-\mathrm{msl}$.

- From C-CG-D-00010 the center grade break in the 488-4D closure cap is $162.35 \mathrm{ft}-\mathrm{msl}$.

- From C-CG-D-00017 Detail 5 the thickness of the closure cap is $2.55 \mathrm{ft}$ (4 inch topsoil + 20 inch common fill +0.3 inch GDL +0.3 inch GCL +6 inch common fill $=30.6$ inches $(2.55 \mathrm{ft}))$.

- $\quad$ Maximum fly ash thickness $=(162.35-2.55)-114=45.8 \mathrm{ft}$ (549.6 inches)

- HELP model default waste \#30 provides the following HELP model default values for highdensity electric plant coal fly ash:

- $\quad$ Saturated hydraulic conductivity $=5.0 \mathrm{E}-05 \mathrm{~cm} / \mathrm{s}$

- $\quad$ Total porosity $(\eta)=0.541$

- $\quad$ Field capacity $=0.187$

- $\quad$ Wilting point $=0.047$

- The above values will be used to represent the fly ash in 488-4D

All 488-4D HELP model design input data has been summarized in Table 2-3 for the base case involving the use of a GCL barrier layer. Multiple HELP model runs were made to evaluate both the nominal and corner slopes and a range of GCL saturated hydraulic conductivities (i.e. 5E-09 (i.e. manufacturer's maximum value), 1E-08, 1E-07, 1E-06, and 1E-05 cm/s (i.e. SCDHEC required saturated hydraulic conductivity for the barrier layer)). All 488-4D HELP model design input data has been summarized in Table 2-4 for the alternative case involving the use of a HDPE geomembrane barrier layer rather than a GCL barrier layer. Table 2-3 and Table 2-4 were used to produce the following HELP model input decks (output file name also provided): 


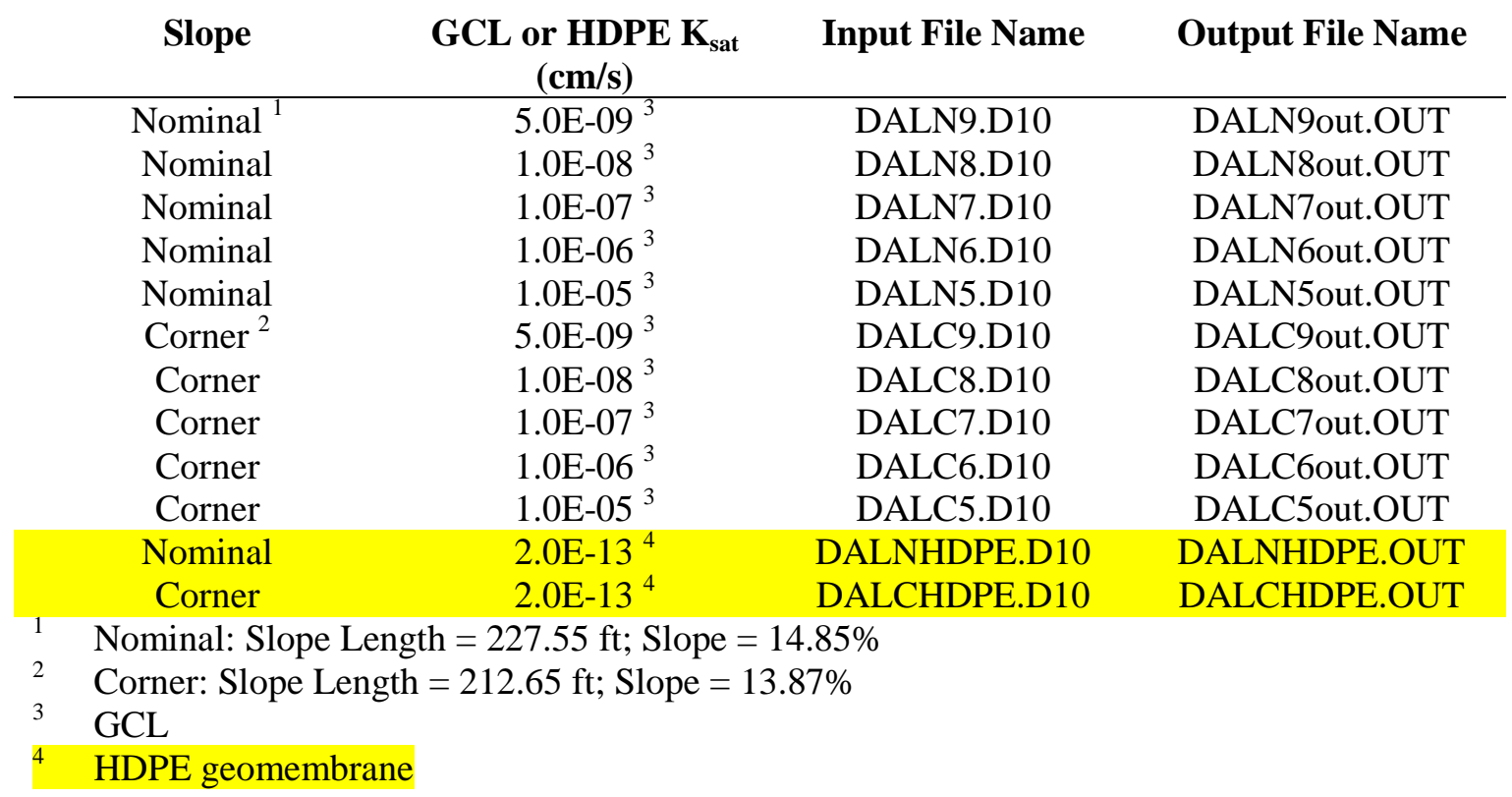


Table 2-3. HELP Model Input Data for D-Area Ash Landfill Base Case Closure Cap with GCL Barrier Layer (as-built):

\begin{tabular}{|c|c|c|c|c|c|c|c|c|c|c|c|}
\hline \multicolumn{7}{|c|}{ Input Parameter (HELP Model Query) } & \multicolumn{5}{|c|}{ Generic Input Parameter Value } \\
\hline \multicolumn{7}{|c|}{ Landfill area $=$} & \multicolumn{5}{|c|}{21.5 acres } \\
\hline \multicolumn{7}{|c|}{ Percent of area where runoff is possible $=$} & \multicolumn{5}{|c|}{$100 \%$} \\
\hline \multicolumn{7}{|c|}{ Do you want to specify initial moisture storage? $(\mathrm{Y} / \mathrm{N})$} & \multicolumn{5}{|c|}{$\mathrm{Y}$} \\
\hline \multicolumn{7}{|c|}{ Amount of water or snow on surface $=$} & \multicolumn{5}{|c|}{0 inches } \\
\hline \multicolumn{7}{|c|}{ CN Input Parameter (HELP Model Query) } & \multicolumn{5}{|c|}{ CN Input Parameter Value } \\
\hline \multicolumn{7}{|c|}{ Slope $=$} & \multicolumn{5}{|c|}{$14.85 \%^{1}$} \\
\hline \multicolumn{7}{|c|}{ Slope length $=$} & \multicolumn{5}{|c|}{$227.55 \mathrm{ft}^{1}$} \\
\hline \multicolumn{7}{|c|}{ Soil Texture $=$} & \multicolumn{5}{|c|}{4 (HELP model default soil texture) } \\
\hline \multicolumn{7}{|c|}{ Vegetation $=$} & \multicolumn{5}{|c|}{4 (i.e., a good stand of grass) } \\
\hline \multicolumn{12}{|c|}{ HELP Model Computed Curve Number $=54.2^{1}$} \\
\hline \multicolumn{3}{|l|}{ Layer } & \multicolumn{5}{|c|}{ Layer Number } & \multicolumn{4}{|c|}{ Layer Type } \\
\hline Topsoil & & & 1 & & & & & 1 (vertic & al percolati & n lay & \\
\hline Upper $C$ & Common & Fill & 2 & & & & & 1 (vertic & al percolati & on laj & \\
\hline GDL & & & 3 & & & & & 2 (latera & drainage & yer) & \\
\hline GCL & & & 4 & & & & & 3 (barrie & r soil liner) & & \\
\hline Lower & Commor & Fill & 5 & & & & & 1 (vertic & al percolati & n lay & \\
\hline Fly Ash & & & 6 & & & & & 1 (vertic & al percolati & on laj & \\
\hline $\begin{array}{l}\text { Layer } \\
\#\end{array}$ & \begin{tabular}{|l} 
Layer \\
Type
\end{tabular} & $\begin{array}{l}\text { Layer } \\
\text { Thickness } \\
\text { (in) }\end{array}$ & & $\begin{array}{l}\text { oil } \\
\text { exture } \\
\text { o. } \\
\end{array}$ & $\begin{array}{l}\text { To } \\
\text { Po } \\
\text { (V }\end{array}$ & $\begin{array}{l}\text { l } \\
\text { sity } \\
\text { /Vol) }\end{array}$ & $\begin{array}{l}\text { Fiel } \\
\text { Cap } \\
\text { (Vo }\end{array}$ & $\begin{array}{l}\text { d } \\
\text { acity } \\
\text { l/Vol) }\end{array}$ & \begin{tabular}{|l|} 
Wilting \\
Point \\
(Vol/Vol) \\
\end{tabular} & & $\begin{array}{l}\text { Initial } \\
\text { Moisture } 2 \\
\text { (Vol/Vol) }\end{array}$ \\
\hline 1 & 1 & 4 & & & 0.3 & & 0.10 & & 0.047 & & 0.109 \\
\hline 2 & 1 & 20 & & & 0.3 & & 0.24 & & 0.202 & & 0.247 \\
\hline 3 & 2 & 0.3 & & & 0.8 & & 0.01 & & 0.005 & & 0.010 \\
\hline 4 & 3 & 0.3 & & & 0.7 & & 0.74 & & 0.400 & & 0.750 \\
\hline 5 & 1 & 6 & & & 0.3 & & 0.24 & & 0.202 & & 0.247 \\
\hline 6 & 1 & 549.6 & & & 0.5 & & 0.18 & & 0.047 & & 0.187 \\
\hline $\begin{array}{l}\text { Layer } \\
\#\end{array}$ & $\begin{array}{l}\text { Layer } \\
\text { Type }\end{array}$ & $\begin{array}{l}\text { Sat. Hyd. } \\
\text { Conductivit } \\
\text { (cm/sec) }\end{array}$ & & $\begin{array}{l}\text { Drainz } \\
\text { Lengtl } \\
\text { (ft) }\end{array}$ & & \begin{tabular}{|l}
$\begin{array}{l}\text { Drain } \\
\text { Slope } \\
\text { (\%) }\end{array}$ \\
\end{tabular} & & $\begin{array}{l}\text { Leachate } \\
\text { Recirc. } \\
(\%)\end{array}$ & \begin{tabular}{l|l} 
Recir \\
Layeı \\
$(\#)$
\end{tabular} & & \begin{tabular}{|l|} 
Subsurface \\
Inflow \\
(in/yr)
\end{tabular} \\
\hline 1 & 1 & $3.1 \mathrm{E}-03$ & & & & & & & & & \\
\hline 2 & 1 & $4.4 \mathrm{E}-05$ & & & & & & & & & \\
\hline 3 & 2 & 11.8 & & 227.5 & & 14.85 & & & & & \\
\hline 4 & 3 & $5 \mathrm{E}-09^{3}$ & & & & & & & & & \\
\hline 5 & 1 & 4.4E-05 & & & & & & & & & \\
\hline 6 & 1 & $5.0 \mathrm{E}-05$ & & & & & & & & & \\
\hline $\begin{array}{l}\text { Layer } \\
\#\end{array}$ & $\begin{array}{l}\text { Layer } \\
\text { Type }\end{array}$ & $\begin{array}{l}\text { Geomembra } \\
\text { Pinhole Den } \\
\text { (\#/acre) }\end{array}$ & & & $\begin{array}{l}\text { reom } \\
\text { efec } \\
\text { t/acr }\end{array}$ & nbrane I & & $\begin{array}{l}\text { Geomem } \\
\text { Placeme }\end{array}$ & $\begin{array}{l}\text { brane } \\
\text { t Quality }\end{array}$ & $\begin{array}{l}\text { Ge } \\
\text { Tra } \\
\text { (cm }\end{array}$ & $\begin{array}{l}\text { textile } \\
\text { nsmissivity } \\
\left.{ }^{2} / \mathrm{sec}\right)\end{array}$ \\
\hline 1 & 1 & & & & & & & & & & \\
\hline 2 & 1 & & & & & & & & & & \\
\hline 3 & 2 & & & & & & & & & & \\
\hline 4 & 3 & & & & & & & & & & \\
\hline 5 & 1 & & & & & & & & & & \\
\hline 6 & 1 & & & & & & & & & & \\
\hline
\end{tabular}

Notes to Table 2-3:

- The lack of values in the table for particular parameters in particular layers denotes that no HELP model input was required for that parameter in that layer. No data are missing from the table.

1 Values associated with the nominal (Slope Length $=227.55 \mathrm{ft}$; Slope $=14.85 \%$ ) and corner (Slope Length $=212.65 \mathrm{ft}$; Slope $=13.87 \%$ ) flow paths will be used within the HELP model runs. HELP Model Computed Curve Number is 54.2 and 54.4 for the nominal and corner slopes. 
2 Initial moisture is set at field capacity for vertical percolation and lateral drainage layers and at total porosity for barrier soil liners.

3 The saturated hydraulic conductivity of the GCL (Bentomat ${ }^{\circledR}$ SDN) has been varied using the following values: $5 \mathrm{E}-09 \mathrm{~cm} / \mathrm{s}, 1 \mathrm{E}-08 \mathrm{~cm} / \mathrm{s}, 1 \mathrm{E}-07 \mathrm{~cm} / \mathrm{s}, 1 \mathrm{E}-06 \mathrm{~cm} / \mathrm{s}$, and $1 \mathrm{E}-05 \mathrm{~cm} / \mathrm{s}$.

Table 2-4. HELP Model Input Data for D-Area Ash Landfill Alternative Case Closure Cap with HDPE Geomembrane Barrier Layer (as-built):

\begin{tabular}{|c|c|c|c|c|c|c|c|c|c|c|c|}
\hline \multicolumn{7}{|c|}{ Input Parameter (HELP Model Query) } & \multicolumn{5}{|c|}{ Generic Input Parameter Value } \\
\hline \multicolumn{7}{|c|}{ Landfill area $=$} & \multicolumn{5}{|c|}{21.5 acres } \\
\hline \multicolumn{7}{|c|}{ Percent of area where runoff is possible $=$} & \multicolumn{5}{|c|}{$100 \%$} \\
\hline \multicolumn{7}{|c|}{ Do you want to specify initial moisture storage? $(\mathrm{Y} / \mathrm{N})$} & \multicolumn{5}{|c|}{$\mathrm{Y}$} \\
\hline \multicolumn{7}{|c|}{ Amount of water or snow on surface $=$} & \multicolumn{5}{|c|}{0 inches } \\
\hline \multicolumn{7}{|c|}{ CN Input Parameter (HELP Model Query) } & \multicolumn{5}{|c|}{ CN Input Parameter Value } \\
\hline \multicolumn{7}{|c|}{ Slope $=$} & \multicolumn{5}{|c|}{$14.85 \%^{1}$} \\
\hline \multicolumn{7}{|c|}{ Slope length $=$} & \multicolumn{5}{|c|}{$227.55 \mathrm{ft}^{1}$} \\
\hline \multicolumn{7}{|c|}{ Soil Texture $=$} & \multicolumn{5}{|c|}{4 (HELP model default soil texture) } \\
\hline \multicolumn{7}{|c|}{ Vegetation $=$} & \multicolumn{5}{|c|}{4 (i.e., a good stand of grass) } \\
\hline \multicolumn{12}{|c|}{ HELP Model Computed Curve Number $=54.2^{1}$} \\
\hline \multicolumn{3}{|c|}{ Layer } & \multicolumn{5}{|c|}{ Layer Number } & \multicolumn{4}{|c|}{ Layer Type } \\
\hline Topsoi & & & 1 & & & & & 1 (vertic & al percolati & n lay & \\
\hline Upper & Common & Fill & 2 & & & & & 1 (vertic & al percolati & n lay & \\
\hline GDL & & & 3 & & & & & 2 (latera & drainage l & yer) & \\
\hline HDPE & Geomem & rrane & 4 & & & & & 4 (geom & embrane lir & & \\
\hline Lower & Commor & Fill & 5 & & & & & 1 (vertic & al percolati & on lay & \\
\hline Fly As & & & 6 & & & & & 1 (vertic & al percolati & n lay & \\
\hline $\begin{array}{l}\text { Layer } \\
\#\end{array}$ & $\begin{array}{l}\text { Layer } \\
\text { Type }\end{array}$ & $\begin{array}{l}\text { Layer } \\
\text { Thickness } \\
\text { (in) }\end{array}$ & & $\begin{array}{l}\text { il } \\
\text { exture } \\
\text { o. }\end{array}$ & $\begin{array}{l}\text { Tot } \\
\text { Por } \\
\text { (Vo }\end{array}$ & $\begin{array}{l}\text { sity } \\
\text { Vol) }\end{array}$ & $\begin{array}{l}\text { Fiel } \\
\text { Cap } \\
\text { (Vo }\end{array}$ & $\begin{array}{l}\text { acity } \\
\text { /Vol) }\end{array}$ & $\begin{array}{l}\text { Wilting } \\
\text { Point } \\
\text { (Vol/Vol) }\end{array}$ & & $\begin{array}{l}\text { Initial } \\
\text { Moisture } 2 \\
\text { (Vol/Vol) }\end{array}$ \\
\hline 1 & 1 & 4 & & & 0.3 & & 0.10 & & 0.047 & & 0.109 \\
\hline 2 & 1 & 20 & & & 0.3 & & 0.2 & & 0.202 & & 0.247 \\
\hline 3 & 2 & 0.3 & & & 0.8 & & 0.01 & & 0.005 & & 0.010 \\
\hline 4 & 4 & 0.06 & & & & & & & & & \\
\hline 5 & 1 & 6 & & & 0.3 & & 0.2 & & 0.202 & & 0.247 \\
\hline 6 & 1 & 549.6 & & & 0.5 & & 0.18 & & 0.047 & & 0.187 \\
\hline $\begin{array}{l}\text { Layer } \\
\#\end{array}$ & $\begin{array}{l}\text { Layer } \\
\text { Type }\end{array}$ & $\begin{array}{l}\text { Sat. Hyd. } \\
\text { Conductivity } \\
\text { (cm/sec) }\end{array}$ & & $\begin{array}{l}\text { Draina } \\
\text { Length } \\
\text { (ft) }\end{array}$ & & $\begin{array}{l}\text { Drain } \\
\text { Slope } \\
(\%)\end{array}$ & & $\begin{array}{l}\text { Leachate } \\
\text { Recirc. } \\
\text { (\%) }\end{array}$ & \begin{tabular}{l|l} 
Recir \\
Layer \\
$(\#)$
\end{tabular} & & \begin{tabular}{|l|} 
Subsurface \\
Inflow \\
(in/yr)
\end{tabular} \\
\hline 1 & 1 & $3.1 \mathrm{E}-03$ & & & & & & & & & \\
\hline 2 & 1 & $4.4 \mathrm{E}-05$ & & & & & & & & & \\
\hline 3 & 2 & 11.8 & & 227.55 & & $14.85^{1}$ & & & & & \\
\hline 4 & 4 & $2.0 \mathrm{E}-13$ & & & & & & & & & \\
\hline 5 & 1 & $4.4 \mathrm{E}-05$ & & & & & & & & & \\
\hline 6 & 1 & $5.0 \mathrm{E}-05$ & & & & & & & & & \\
\hline $\begin{array}{l}\text { Layer } \\
\#\end{array}$ & $\begin{array}{l}\text { Layer } \\
\text { Type }\end{array}$ & $\begin{array}{l}\text { Geomembrar } \\
\text { Pinhole Den } \\
\text { (\#/acre) }\end{array}$ & & & $\begin{array}{l}\text { eome } \\
\text { efect } \\
\text { t/acre }\end{array}$ & brane Ir & & $\begin{array}{l}\text { Geomem } \\
\text { Placemei }\end{array}$ & $\begin{array}{l}\text { brane } \\
\text { t Quality }\end{array}$ & $\begin{array}{l}\text { Gec } \\
\text { Tra } \\
(\mathrm{cm}\end{array}$ & $\begin{array}{l}\text { textile } \\
\text { nsmissivity } \\
\left.1^{2} / \mathrm{sec}\right)\end{array}$ \\
\hline 1 & 1 & & & & & & & & & & \\
\hline 2 & 1 & & & & & & & & & & \\
\hline 3 & 2 & & & & & & & & & & \\
\hline 4 & 4 & 1 & & 4 & & & & 3 (Good) & & & \\
\hline 5 & 1 & & & & & & & & & & \\
\hline 6 & 1 & & & & & & & & & & \\
\hline
\end{tabular}


Notes to Table 2-4:

- The lack of values in the table for particular parameters in particular layers denotes that no HELP model input was required for that parameter in that layer. No data are missing from the table.

1 Values associated with the nominal (Slope Length $=227.55 \mathrm{ft}$; Slope $=14.85 \%$ ) and corner (Slope Length $=212.65 \mathrm{ft}$; Slope $=13.87 \%$ ) flow paths will be used within the HELP model runs. HELP Model Computed Curve Number is 54.2 and 54.4 for the nominal and corner slopes.

2 Initial moisture is set at field capacity for vertical percolation and lateral drainage layers and at total porosity for barrier soil liners.

\subsection{Quality Assurance}

The software quality assurance plan for the use of the HELP model is documented in Phifer 2006. A technical review of this work was performed consistent with the E7 Manual, procedure 2.60 as outlined in SRNL Technical Report Design Check Guidelines (WSRC 2004).

\subsection{Results and Conclusions}

The HELP model has been run for the 488-4D closure cap as outlined in Section 2.0 for both the base case (GCL barrier layer) and an alternative case (HDPE geomembrane barrier layer), and both annual average and daily output data has been generated. Table 3-1 provides a summary of the results. As shown in Table 3-1 infiltration increases and lateral drainage decreases as the assumed saturated hydraulic conductivity of the GCL increases for the base case. Changing the assumed saturated hydraulic conductivity of the GCL does not impact the runoff or evapotranspiration, because these two parameters are primarily affected by the properties of the topsoil and upper common fill and the slope and slope length. Also as seen in Table 3-1 there is very little difference between the associated nominal and corner runs. A comparison of the Table 3-1 alternative case (HDPE geomembrane barrier layer) results with the base case (GCL barrier layer with a saturated hydraulic conductivity of $5.0 \mathrm{E}-09 \mathrm{~cm} / \mathrm{s}$ ) results demonstrates the following:

- Both cases resulted in the same runoff and evapotranspiration values.

- Alternative case (HDPE geomembrane barrier layer) resulted in slightly more lateral drainage and slightly less infiltration.

- The heads for both cases were either the same or essentially the same.

The SCDHEC design storm event is a 25-year return period, 24-hour accumulation period storm. Based upon Weber 1998 Table XIX, a 25-year, 24-hour storm event is 6.1 inches. For landfills SCDHEC requires that no more than a 12 inch head develop on top of the barrier layer (saturated hydraulic conductivity of no more than $1.0 \mathrm{E}-05 \mathrm{~cm} / \mathrm{s}$ ) in association with a 25-year, 24-hour storm event. As seen in Table 3-1 the greatest peak daily head on top of the barrier layer (either GCL or HDPE) for any of the runs made was 0.079 inches associated with a peak daily precipitation of 6.16 inches. This is well below the SCDHEC limit of 12 inches. 
Table 3-1. 488-4D HELP Model Summary Output

\begin{tabular}{|c|c|c|c|c|c|c|c|c|c|c|}
\hline Slope & $\begin{array}{c}\text { GCL or } \\
\text { HDPE } \\
K_{\text {sat }} 1 \\
(\mathrm{~cm} / \mathrm{s})\end{array}$ & $\begin{array}{c}\text { Precip }^{2} \\
\text { (in/yr) }\end{array}$ & $\begin{array}{l}\text { Runoff } \\
\text { (in/yr) }\end{array}$ & $\begin{array}{c}\text { ET }^{3} \\
\text { (in/yr) }\end{array}$ & $\begin{array}{c}\text { Lateral } \\
\text { Drainage } \\
\text { (in/yr) }\end{array}$ & $\begin{array}{c}\text { Infiltration } \\
\text { thru GCL } \\
\text { (in/yr) }\end{array}$ & $\begin{array}{c}\text { Average } \\
\text { Annual } \\
\text { Head on } \\
\text { GCL } \\
\text { (in) }\end{array}$ & $\begin{array}{c}\text { Peak } \\
\text { Daily } \\
\text { Precip } \\
\text { (in/day) }\end{array}$ & $\begin{array}{c}\text { Peak } \\
\text { Average } \\
\text { Daily } \\
\text { Head on } \\
\text { GCL } \\
\text { (in) } \\
\end{array}$ & $\begin{array}{c}\text { Peak } \\
\text { Maximum } \\
\text { Daily } \\
\text { Head on } \\
\text { GCL } \\
\text { (in) } \\
\end{array}$ \\
\hline Nominal & $5.0 \mathrm{E}-09^{4}$ & 47.93 & 0.704 & 19.793 & 27.39673 & 0.02256 & 0.002 & 6.16 & 0.037 & 0.079 \\
\hline Nominal & $1.0 \mathrm{E}-08^{4}$ & 47.93 & 0.704 & 19.793 & 27.37437 & 0.04490 & 0.002 & 6.16 & 0.037 & 0.070 \\
\hline Nominal & $1.0 \mathrm{E}-07^{4}$ & 47.93 & 0.704 & 19.793 & 26.99414 & 0.42513 & 0.002 & 6.16 & 0.037 & 0.055 \\
\hline Nominal & $1.0 \mathrm{E}-06^{4}$ & 47.93 & 0.704 & 19.793 & 24.62621 & 2.79307 & 0.002 & 6.16 & 0.037 & 0.076 \\
\hline Nominal & $1.0 \mathrm{E}-05^{4}$ & 47.93 & 0.704 & 19.793 & 17.30483 & 10.11440 & 0.002 & 6.16 & 0.037 & 0.051 \\
\hline Corner & $5.0 \mathrm{E}-09^{4}$ & 47.93 & 0.702 & 19.907 & 27.28500 & 0.02269 & 0.002 & 6.16 & 0.037 & 0.059 \\
\hline Corner & $1.0 \mathrm{E}-08^{4}$ & 47.93 & 0.702 & 19.907 & 27.26259 & 0.04510 & 0.002 & 6.16 & 0.037 & 0.078 \\
\hline Corner & $1.0 \mathrm{E}-07^{4}$ & 47.93 & 0.702 & 19.907 & 26.88053 & 0.42716 & 0.002 & 6.16 & 0.037 & 0.070 \\
\hline Corner & $1.0 \mathrm{E}-06^{4}$ & 47.93 & 0.702 & 19.907 & 24.50455 & 2.80313 & 0.002 & 6.16 & 0.037 & 0.077 \\
\hline Corner & $1.0 \mathrm{E}-05^{4}$ & 47.93 & 0.702 & 19.907 & 17.18225 & 10.12538 & 0.002 & 6.16 & 0.037 & 0.057 \\
\hline Normal & $2.0 \mathrm{E}-13^{5}$ & 47.93 & 0.704 & 19.793 & 27.41905 & 0.00023 & 0.002 & 6.16 & 0.037 & 0.054 \\
\hline Corner & $2.0 \mathrm{E}-13^{5}$ & 47.93 & 0.702 & 19.907 & 27.30745 & 0.00023 & 0.002 & 6.16 & 0.037 & 0.070 \\
\hline
\end{tabular}

$\mathrm{K}_{\mathrm{sat}}=$ saturated hydraulic conductivity

Precip = precipitation

ET = evapotranspiration

GCL barrier layer

5 HDPE geomembrane barrier layer 
SRNL-STI-2014-00392

Revision 1

\subsection{References}

Agru 2013. 300 mil Geocomposite, Agru America, Inc., Georgetown, SC. (http://www.agruamerica.com/wp-content/uploads/2013/09/300-mil-GeocompositeDataSheets.pdf)

ASTM D5268 - 13, Standard Specification for Topsoil Used for Landscaping Purposes, ASTM International, West Conshohocken, PA. July 2013.

C-CG-D-00010, D-Area Expanded Operable Unit 488-4D Ash Landfill Closure 488-4D Final Grading Plan (Sheet 1 of 2).

C-CG-D-00011, D-Area Expanded Operable Unit 488-4D Ash Landfill Closure 488-4D Final Grading Plan (Sheet 2 of 2).

C-CG-D-00012, D-Area Expanded Operable Unit 488-4D Ash Landfill Closure Cross Sections (Sheet 1 of 2).

C-CG-D-00017, D-Area Expanded Operable Unit 488-4D Ash Landfill Closure Final Cover Details (Sheet 1 of 2).

C-SPP-D-00002, Rev. 0, 488-4D Ash Landfill Closure Specifications, June 3, 2014.

CETCO 2009. Bentomat SDN Certified Properties, TR 401-BMSDN, CETCO Lining Technologies, Hoffman Estates, Illinois. March 2009.

(http://lining.cetco.com/DesktopModules/Bring2mind/DMX/Download.aspx?EntryId=11133\&Command =Core_Download\&language $=$ en-US\&PortalId=12\&TabId=1476)

E7 Manual - Conduct of Engineering, Procedure 2.60 - Technical Reviews, Rev. 14, April 24, 2012.

GRI 2013. Standard Guide for "Design Considerations for Geosynthetic Clay Liners (GCLs) in Various Applications, GRI-GCL5, Revision 1, Geosynthetic Research Institute, Folsom, PA. January 9, 2013.

Koerner, R. M. 1990. Designing with Geosynthetics, Second Edition. Prentice Hall, Englewood Cliffs, New Jersey. 1990.

Phifer, M. A. 2006. Software Quality Assurance Plan for the Hydrologic Evaluation of Landfill Performance (HELP) Model, Q-SQA-A-00005, Revision 0. Washington Savannah River Company, Aiken, SC. October 2006.

Phifer, M. A., Jones, W. E., Nelson, E. A., Denham, M. E., Lewis, M. R., and Shine, E. P. 2007. FTF Closure Cap Concept and Infiltration Estimates, WSRC-STI-2007-00184 OUO, Rev 2. Savannah River National Laboratory, Aiken, SC. October 15, 2007.

Schroeder, P. R., Lloyd, C. M., Zappi, P. A., and Aziz, N. M. 1994a. The Hydrologic Evaluation of Landfill Performance (HELP) Model User's Guide for Version 3. EPA/600/R-94/168a. Office of Research and Development, United States Environmental Protection Agency (EPA), Cincinnati, Ohio. September 1994.

Schroeder, P. R., Dozier, T. S., Zappi, P. A., McEnroe, B. M., Sjostrom, J. W., and Peyton, R. L. 1994b. The Hydrologic Evaluation of Landfill Performance (HELP) Engineering Documentation for Version 3. 
EPA/600/R-94/168b. Office of Research and Development, United States Environmental Protection Agency (EPA), Cincinnati, Ohio. September 1994.

Schroeder, P. R. and Peyton, R. L. 1987a. Verification of the Lateral Drainage Component of the HELP Model Using Physical Models. EPA/600/2-87/049. Office of Research and Development, United States Environmental Protection Agency (EPA), Cincinnati, Ohio. July 1987.

Schroeder, P. R. and Peyton, R. L. 1987b. Verification of the Hydrologic Evaluation of Landfill Performance (HELP) Model Using Field Data. EPA/600/2-87/050. Office of Research and Development, United States Environmental Protection Agency (EPA), Cincinnati, Ohio. July 1987.

Weber, A. H. 1998. Tornado, Maximum Wind Gust, and Extreme Rainfall Event Recurrence Frequencies at the Savannah River Site, WSRC-TR-98-00329, Westinghouse Savannah River Company, Aiken, SC. September 1998.

WSRC 2004. Savannah River National Laboratory Technical Report Design Check Guidelines, WSRCIM-2002-00011, Revision 2, Westinghouse Savannah River Company, Aiken, SC. August 2004

Yu, A. D., Langton, C. A., and Serrato, M. G. 1993. Physical Properties Measurement Program (U), WSRC-RP-93-894. Westinghouse Savannah River Company, Aiken, SC. June 30, 1993. 
SRNL-STI-2014-00392

Revision 1

Intentionally Blank 


\section{Distribution:}

R. S. Aylward, 773-42A

D. D. Brett, 730-4B

B. T. Butcher, 773-43A

D. A. Crowley, 773-43A

T. F. Gaughan, 730-4B

J. C. Griffin, 773-A

J. M. Lovekamp, 730-2B

T. O. Oliver, 773-42A

M. A. Phifer, 773-42A

F. C. Sappington, 730-4B

E. P. Schiefer, 730-4B

R. R. Seitz, 773-43A

F. G. Smith, 703-41A

R. P. Socha, 730-4B

T. S. Whiteside, 773-42A

C. Wilson (1 file copy \& 1 electronic copy), 773-43A 